\title{
Intra-articular guanethidine injection for resistant shoulder pain: a preliminary double blind study of a novel approach
}

\author{
Kamel Gado, Paul Emery
}

\begin{abstract}
Objective-To study the effect of intraarticular (IA) sympathetic blockade for the relief of resistant shoulder pain. Methods-Eighteen patients with shoulder pain resistant to conventional treatment were allocated randomly to two groups, to receive either IA guanethidine $20 \mathrm{mg}$ or IA saline. They were assessed for pain, and range of active movements, before injection and at one, four, and eight weeks after injection.

Results-There were no significant differences between groups, but the group receiving guanethidine showed greater improvement in pain relief at all three follow up visits compared with those receiving placebo $(9 \% v 7 \%$ at one week; $15 \% v 6 \%$ at four weeks and $36 \% v 16 \%$ at eight weeks). The improvement reached significance $(p<0.05)$ at the eight week visit compared with baseline. The range of movement was not significantly improved in either group.

Conclusion-The results suggest that IA guanethidine produced measurable improvement in resistant shoulder pain and that further studies of this novel approach are indicated.
\end{abstract}

(Ann Rheum Dis 1996; 55: 199-201)

Shoulder pain is one of the most common medical problems. In the USA, Kelsey reported in $1982^{1}$ an incidence of 1 million cases in 1967. In the United Kingdom, data from the Department of Health and Social Security suggest an incidence of 1 in $170 .^{2}$ Several investigators in both the United Kingdom and Scandinavia have reported a prevalence of about $20 \%$ in geriatric populations. $^{34}$ The most common causes of shoulder pain (as a result of shoulder disease) are rheumatoid arthritis (RA), frozen shoulder, rotator cuff tendonitis, and osteoarthritis (OA). Although conventional treatment, which includes intra-articular (IA) injections of steroid, physiotherapy, suprascapular nerve block $^{5}$ and various other forms of pain relief modalities, is effective in the majority of patients, a painful stiff shoulder remains a common cause of prolonged disability for many patients.

Considerable interest has been shown in the role of the sympathetic nervous system (SNS) in musculoskeletal pain transmission in the pathogenesis of inflammation. Studies have shown that substances blocking the SNS (namely guanethidine and reserpine) were able to reduce inflammation and joint injury in animals in an experimental model of arthritis ${ }^{7}$ and patients with RA. ${ }^{8}$ Sympathetic innervation of the shoulder in humans was first reported by Gardner in $1948 ;{ }^{9}$ anatomical studies of animal models have provided further evidence of sympathetic innervation of the elbow and knee. ${ }^{10}$ Guanethidine is the drug used most commonly as an SNS blocker, and has been shown to deplete peripheral stores of catecholamines. ${ }^{8}$ Intravenous (IV) guanethidine, used regionally, has been shown to achieve measurable pain relief and an increase in pinch strength. ${ }^{10}$ In 1992, Butler-Manuel et al used scintigraphy to show that anterior knee pain was sympathetically mediated, and they provided both subjective and objective evidence of improvement after lumbar sympathetic blockade. ${ }^{11}$ Reflex sympathetic dystrophy of either shoulder or knee joints is another well known example of the relationship of SNS and chronic pain syndromes. ${ }^{12} \mathrm{Re}-$ gional sympathetic blockade using guanethidine is now a standard treatment in pain clinics for cases of reflex sympathetic dystrophy of the lower limbs. ${ }^{13}$

It is possible that at least a component of chronic shoulder pain is sympathetically mediated. The aim of this study was to investigate the efficacy of a new route of injection of guanethidine (namely IA), in alleviating shoulder pain resistant to other treatments.

\section{Patients and methods}

PATIENT POPULATION

The study was approved by the South Birmingham Health Authority Ethical Committee. Eighteen patients who agreed to participate in the study and gave informed consent were recruited from the shoulder clinics of Queen Elizabeth and Selly Oak Hospitals, Birmingham, United Kingdom. They comprised six patients with RA (American College of Rheumatology criteria ${ }^{14}$ ), five patients with OA (diagnosed according to both clinical and radiological criteria), five patients with frozen shoulder (according to the Cyriax criteria ${ }^{15}$ - that is, painful limitation of all shoulder movement with external rotation of less than $50 \%$ ), one patient with rotator cuff tendonitis that was diagnosed by painful limitation of active abduction with positive 
painful arc sign, and one patient with psoriatic arthritis diagnosed clinically.

All patients suffered from persistent joint pain on movement with or without rest pain and had failed the standard shoulder protocol which included sequential treatment with an IA steroid (given subacromially and posteriorally), followed by suprascapular nerve block and physiotherapy. All patients had received a nerve block in the previous three months. Patients with fractures, dislocation of the humerus or cervical spondylosis were excluded, as were those with any sensory symptoms or signs in the affected arm, or radiation of pain to the neck. All patients were receiving analgesics, all patients except those with $\mathrm{OA}$ were also receiving anti-inflammatory drugs, and patients with RA were also taking disease modifying medication. No alteration of the dose of medication was allowed during the period of study.

EXPERIMENTAL PROCEDURE

All the patients were blinded as to the treatment they received. This was selected from preshuffled randomisation cards by the shoulder clinic nurse who filled the syringe. Patients received either $20 \mathrm{mg}$ guanethidine (2 $\mathrm{ml}$ ) or $0.9 \%$ saline $2 \mathrm{ml}$ by the posterior IA approach. Injections and assessment were performed by a physician blind to the treatment. Patients were assessed before injection and at one, four, and eight weeks after injection. Assessments included the degree of pain on a $10 \mathrm{~cm}$ vertical visual analogue scale (VASP) and the range of active movements of flexion, extension, abduction, and external rotation, recorded with a goniometer. Patients were allowed to rest on a couch for one hour after the procedure. As dictated by the ethics committee, the patients who received placebo drug had the option of receiving active medication at the end of the study; six patients in the placebo group chose to do this and were reassessed at eight weeks after active injection.

STATISTICAL ANALYSIS

Statistical analysis was performed comparing the follow up values with the base line values (visit 0) using Student's $t$ test.

\section{Results}

No side effects were recorded during the course of study from either injections; in particular, there was no significant hypotension.

GUANETHIDINE GROUP

The diagnoses of the patients who received IA guanethidine were OA (four), RA (two), frozen shoulder (two), and psoriatic arthritis (one). For these patients, the mean reduction in VASP value at follow up was $8.9 \%, 15.3 \%$, and $35.9 \%$ at the three follow up visits. The difference between follow up and baseline reached significance for the eight week visit $(p<0.05)$ (figure). The range of active move-

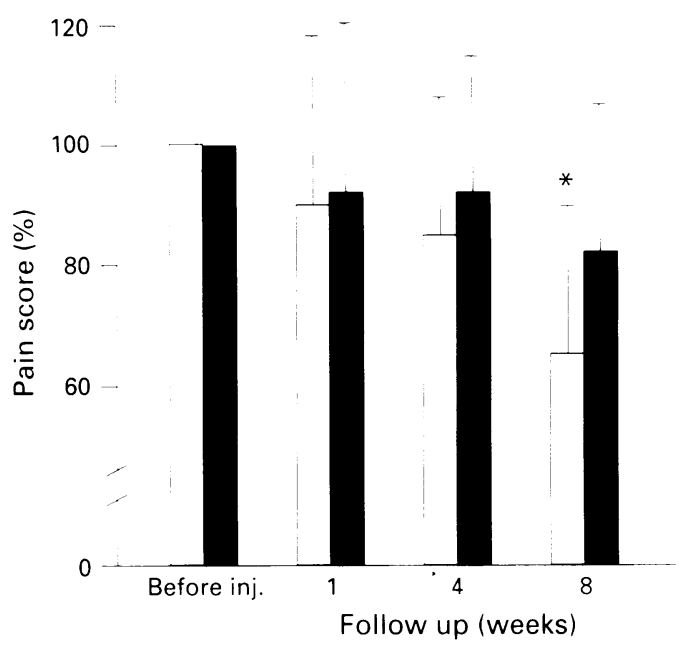

Effect of IA guanethidine ( $\square$ ) or saline ( $\square$ ) on pain score at visit 0 (Before inj.) (which is taken as 100\%) and follow up visits. ${ }^{\star}$ Significant difference $(p<0 \cdot 05)$.

ment showed no significant changes compared with base line (table).

SALINE GROUP

The diagnoses of the patients who received IA saline were RA (four), OA (one), frozen shoulder (three), and rotator cuff tendonitis (one). The reduction in VASP at the three follow up assessments was $8.4 \%, 5.9 \%$, and $16.4 \%$ respectively, none of which were significantly different from base line (figure). Again, there was no statistically significant change in range of active movement (table).

Six patients in the saline group subsequently received IA guanethidine at the end of the study. Four of these, who had OA, RA (two), and frozen shoulder, recorded a marked $(>50 \%)$ reduction in VASP on the follow up visit two months after the end of the study. The results are not included in the statistical analysis as these patients did not form part of the double blind trial.

\section{Discussion}

Guanethidine has been used routinely in the past few years to produce regional blockade of the SNS and relieve the pain of reflex sympathetic dystrophy. This has, in general, been performed as a regional block requiring the application of a proximal tourniquet and administering the drug IV. Our double blind

Range of active movement at visit 0 (before injection) and follow up visits in patients given guanethidine or saline IA

\begin{tabular}{|c|c|c|c|c|}
\hline \multirow[t]{2}{*}{ Group } & \multirow{2}{*}{$\begin{array}{l}\text { Visit } \\
O\end{array}$} & \multicolumn{3}{|c|}{ Follow up visits } \\
\hline & & 1 week & 4 weeks & 8 weeks \\
\hline \multicolumn{5}{|l|}{ Guanethidine } \\
\hline Flexion & $62 \cdot 3(20)$ & $61 \cdot 1(20)$ & $62 \cdot 8(25)$ & $65 \cdot 8(20)$ \\
\hline Extension & $55 \cdot 7(15)$ & $60(17)$ & $57(17)$ & $53.9(17)$ \\
\hline Abduction & $52.9(17)$ & $55(19)$ & $50 \cdot 7(16)$ & $52 \cdot 4(18)$ \\
\hline Ext rotation & $26 \cdot 4(22)$ & $32 \cdot 4(22)$ & $30(21)$ & $30 \cdot 7(20)$ \\
\hline \multicolumn{5}{|l|}{ Saline } \\
\hline Flexion & $68 \cdot 8(24)$ & $68 \cdot 3(34)$ & $69 \cdot 9(25)$ & $77 \cdot 7(26)$ \\
\hline Extension & $51(15)$ & $48 \cdot 4(13)$ & $55 \cdot 3(12)$ & $53 \cdot 8(12)$ \\
\hline Abduction & $57 \cdot 4(17)$ & $58 \cdot 1(14)$ & $60 \cdot 2(19)$ & $55 \cdot 7(12)$ \\
\hline Ext rotation & $25 \cdot 6(12)$ & $33 \cdot 3(19)$ & $27 \cdot 9(17)$ & $39 \cdot 6(18)$ \\
\hline
\end{tabular}

Values are mean (SD) 
study, the first attempt to investigate the efficacy of an IA injection of guanethidine in the relief of resistant shoulder pain, revealed that guanethidine produced a significant improvement at the two month follow up, which was not seen in the saline group. The level of improvement of VASP in the saline group was within the recognised values for placebo group (up to $30 \%$ ). ${ }^{16}$ It was interesting to note that significant $(>50 \%)$ improvement was also seen in four of the six patients who, having failed to respond to placebo, subsequently received IA guanethidine.

A previous study of regional IV guanethidine has shown improvement of their pain in patients with RA. ${ }^{8}$ The effect of guanethidine is presumed to be mediated largely by the depletion of catecholamines from sympathetic postganglionic efferents. Reduced sympathetic outflow would decrease the direct inflammatory effect of catecholamines and the indirect catecholamine stimulation of unmyelinated peptidergic afferents implicated in the inflammatory process. ${ }^{17}$ The fact that all our patients were resistant to IA steroid injection, physiotherapy, and other well known pain relief modalities, made it difficult to compare our results with results of other studies using the previously mentioned modalities. All patients had previously received suprascapular nerve block; we do not know whether this in some way sensitised the patients to respond to guanethidine. The observation of no significant improvement in range of movement in either group was not unexpected, as most of the patients had marked destruction of the shoulder joint radiologically.

The results achieved in this preliminary study suggest that IA guanethidine has a measurable effect in patients with resistant shoulder pain. Despite the relatively small number and heterogenous nature of the patients, a significant improvement was found in the active group and this should be considered encouraging. However, it is clear that a longer term study with a larger number of more homogenous patients is required before the value of this approach can be fully assessed. Whether it is possible to recognise symptoms that indicate SNS involvement, or whether the earlier use of SNS blockade could be more effective, are questions which both require attention. This treatment does, however, appear to offer another option in a group of patients with limited treatment alternatives.

We thank our shoulder clinic nurse, Mrs Kathy Chiswell, for her help throughout the course of this study. This research was sponsored by a grant from the Arthritis and Rheumatism Sponsored by a grant from

1 Kelsey J L. Epidemiology of musculoskeletal disorders. New York: Oxford University Press, 1982; 194

2 Department of Health and Social Security. Morbidity statistics from general practice. Third National Study 1981-1982. London: HMSO.

3 Chard M, Hazleman R, Hazleman B L. Shoulder disorders in the elderly (a community survey). Br f Rheumatol 1989; 28: 21 .

4 Takala J, Siever K A, Klankka T. Rheumatic symptoms in Southwestern Finland. Scand $f$ Rheumatol 1982; 47: 15-29.

5 Emery P, Bowman S, Wedderburn L, Grahame R. Suprascapular nerve block for chronic shoulder pain in rheumatoid arthritis. $B M \mathcal{F}$ 1989; 299: 1079-80.

6 Gado K S H, Emery P. Modified suprascapular nerve block with bupivacaine alone effectively controls chronic shoulder pain in patients with rheumatoid arthritis. Ann Rheum Dis 1993; 52: 215-8.

7 Lotz M, Carson D A, Vaughan J H. Substance P activation of rheumatoid synoviocytes: neural pathway in pathogenesis of arthritis. Science 1987; 235: 893-5.

8 Levine J D, Fye K, Heller P, Basbaum A I, Whiting-O'Keefe Q Clinical response to regional intravenous guanethidine in patients with theumatoid arthritis. $\mathcal{F}$ Rheumatol 1986; 13: patients

9 Gardner E. The innervation of the shoulder joint. Anat Rec 1948; 102: 1-18.

10 Wilberge $M$, Widenfalk $B$. An anatomical study of the origin of sympathetic innervation of the elbow and knee joint in the monkey. Neurosci Lett 1991; 127: 185-8.

11 Butler-Manuel P A, Justin D, Heatley F W. Sympathetically mediated anterior knee pain. Scintigraphy and anaesthetic blockade in 19 patients. Acta Orthop Scand 1992; 63: 90-3.

12 Ogilvie-Harris D, Roscoe M. Reflex sympathetic dystrophy of the knee. F Bone foint Surg Br 1987; 69: 804-6.

13 Hanning-Keff J G. Relief of Sudek's atrophy by regional intravenous guanethidine. Lancet 1977; 1: 1132-3.

14 Arnett F C, Edworthy S M, Block D A, et al. The American Rheumatism Association 1987 Revised criteria for the classification of rheumatoid arthritis. Arthritis Rheum 1988; 31: 315-23.

15 Cyriax J. Diagnosis of soft tissue lesions. In: Textbook of Orthopaedic Medicine. London: Bailliere Tindall, 1982; 129.

16 Williams N E. Current views of the pharmacological management of pain. In: Swerdlow M, ed. The therapy of pain, 1st edn. Lancaster: MTP Press Ltd, 1981; 87.

17 Buck S H, Walsh J H, Yamamura H T. Neuropeptides in sensory neurons. Life Sci 1982; 30: 1857-66. 